\title{
Revisión de estudios de la Escala de Ansiedad y Depresión Hospitalaria (HAD) en muestras españolas
}

\author{
M. Carmen Terol-Cantero*, Víctor Cabrera-Perona, Maite Martín-Aragón
}

Universidad Miguel Hernández Elche

\begin{abstract}
Resumen: Introducción: La escala de Ansiedad y Depresión Hospitalaria (HADS-Hospital Anxiety and Depression Scale, Zigmond y Snaith, 1983) se ha convertido en un popular instrumento clínico diseñado para evaluar ansiedad y depresión, encontrándose evidencias de que aplicado a diversas poblaciones parece tener tan buenas propiedades de detección cómo otros instrumentos. Numerosos trabajos en muestra española comprueban sus propiedades psicométricas y puntos de corte. Nuestro objetivo es unificar los resultados de estudios realizados en muestras españolas para concretar directrices que orienten en la aplicabilidad e interpretación de sus puntuaciones. Método: Revisión bibliográfica. Resultados: Se seleccionaron 15 artículos, a) se confirma que las propiedades psicométricas del HAD en diferentes muestras son óptimas, b) se presenta cierta variabilidad de puntos de corte para cada población y c) determinados ítems muestran débil carga factorial e ítem-test. Discusión: se plantea qué ítems deben reconsiderarse para un HAD abreviado. Se sugiere valorar la escala total de distrés.

Palabras clave: HAD; Hospital Anxiety and Depression Scale; revisión; propiedades psicométricas; puntos de corte.
\end{abstract}

\section{Introducción}

La escala de Ansiedad y Depresión Hospitalaria (HADHospital Anxiety and Depression Scale-HADS, Zigmond y Snaith, 1983) ha sido el instrumento de autoevaluación más usado para detectar malestar emocional (ansiedad y depresión) en poblaciones con enfermedad física. Su índice de citaciones es superior a tres mil (Brennan, Worrall-Davies, McMillan, Gilbody y House, 2009; Tyrer y Methuen, 2007). Es un instrumento corto (14 ítems) que ha mostrado su fiabilidad y validez siendo utilizado tanto para el diagnóstico como para evaluar la gravedad del trastorno (Rivera et al., 2006). Se compone de dos subescalas (HADA: ansiedad y HADD: depresión) de siete ítems cada una con puntuaciones de 0 a 3. Los propios autores recomiendan los puntos de corte originales: ocho para casos posibles y $>10$ para casos probables en ambas subescalas (Zigmond y Snaith, 1983; Bjelland, Dahlb, Haugc y Neckelmann, 2002). En una revisión de 24 estudios, estos puntos de corte, muestran una sensibilidad y especificidad de .80 de promedio en pacientes en atención primaria y servicios hospitalarios (Bjelland et al., 2002). Entre sus ventajas, incluye, entre otras a) su sencillez y brevedad, que aumenta la probabilidad de aceptación para su uso, b) la posibilidad de evaluar ansiedad y depresión con la misma escala c) la exclusión de ítems de carácter somático que pudieran confundirse en la evaluación de pacientes físicos con síntomas como pérdida de apetito y/o energía, fati-

* Dirección para correspondencia [Correspondence address]:

M. Carmen Terol Cantero. Dpto. de Psicología de la Salud. Campus de San Juan. Universidad Miguel Hernández de Elche. 03550 San Juan, Alicante (España).E-mail: macarmen@umh.es
Title: Hospital Anxiety and Depression Scale (HADS) review in Spanish Samples.

Abstract. Introduction: The Hospital Anxiety and Depression Scale (HADS, Zigmond and Snaith, 1983) has become a popular clinical instrument designed to assess anxiety and depression. Evidence of HADS' good screening properties applied to several samples has been found. HADS seems to be as good screening properties as other tools. Different studies in Spanish sample tested its psychometric properties, including also their cut-offs points, specificity and sensitivity. Our aim is to unify the results of studies in Spanish population specifying guidelines regarding their applicability and interpretation of their cut-off points. Material and method: bibliographic review. Results: 15 articles were selected, a) HADS confirms its optimal psychometric properties in different groups, b) HADS shows some variability in proposed cut-off points for different groups, c) A number of items shown weak factor loading and item-test. Discussion: These items should be reconsidered for new proposals of a shortened final scale. We suggest attending at distress total scale.

Key words: HADS; Hospital Anxiety and Depression Scale; review; psychometric properties; cut-off points.

ga, insomnio, cambios en actividad, $\mathrm{u}$ otros trastornos fisiológicos, y d) sus ítems aluden a contenidos especialmente relevantes en contextos de salud, incluyendo respuestas cognitivas, emocionales y comportamentales de la ansiedad y la depresión (Terol et al., 2007).

La versión original del HAD ha sido validada y traducida a numerosas lenguas (francés, italiano, chino, alemán, español, etc.) y utilizada en diversos países y culturas (Abiodun, 1994; Constantini, Musso, y Viterbori, 1999; Fossati y Marzocchi, 2008; Leung, Ho, Kan, Hung y Chen, 1993; Malasi, Mirza y el Islam, 1991; Miklavcic, Snoj, Mlakar y Pregelj, 2008; Mumford, Tareen, Bajwa, Bathi y Karin, 1991; Nortvedt, Riiseb y Sannec, 2006; Spinhoven et al., 1997; Tejero, Guimera, Farré y Peri, 1986; Untas et al., 2009). Con el HAD se ha evaluado ansiedad y depresión tanto en enfermos físicos y mentales como en población general, obteniendo resultados que, en general, apoyan su uso como herramienta útil para detectar distrés psicológico en pacientes no psiquiátricos (Brennan et al., 2009). Entre las muestras de enfermos físicos se incluyen pacientes de atención primaria, cirugía, ginecología, dermatología, cáncer, cardiología, fatiga crónica o VIH, entre otros (Abiodun, 1994; Aylard, Gooding, McKenna y Snaith, 1987; Barczack et al., 1988; Burvill, Anderson, Jamrozik, Stewart-Wynne y Chakera, 1995; ElRufaie, y Absood, 1995; Lewis y Wessely, 1990; López-Roig et al., 2000; McCue, Martin, Buchanan, Rodgers y Scholey, 2003; Moorey et al., 1991; Razavi et al., 1992; Savard, Laberge, Gauthier, Ivers y Bergerson, 1998; Upadhyaya y Stanley, 1997; Visser et al., 1995; entre otros). De entre los trabajos realizados con enfermos mentales, se refieren diagnósticos de Depresión Mayor, Distimia, Fobias, Ansiedad generalizada, o Trastornos del Humor, entre otros (Berard y Ahmed, 
1995; De las Cuevas, García-Estrada y González de Rivera, 1995; Hammer, Sanjeev, Butterworth y Barczack, 1991; Ibáñez y Caro, 1992; Malasi, Mirza y el Islam, 1991; Silverstone, 1994; White, Leach, Sims, Atkinson y Cottrell, 1999). En las muestras de población general, se ha evaluado ansiedad y depresión en adolescentes, en estudiantes universitarios, en adultos y en personas de tercera edad (Abiodun, 1994; Caci et al., 2003; Ibáñez y Caro; 1992; Mumford et al., 1991; Quintana et al., 2003; Spinhoven et al., 1997). Parte de estos trabajos han tratado de establecer si el HAD puede utilizarse como instrumento de screening para detectar trastornos severos de ansiedad y/o depresión, y también si sus subescalas funcionan diferenciando ambos constructos, o quizá, deban considerarse con una única puntuación global de distrés psicológico.

Estas revisiones y los meta-análisis realizados sobre el HAD muestran, sin embargo, cierta heterogeneidad de puntos de corte, sensibilidad y especificidad y estructura factorial (Bjelland et al., 2002; Brennan et al., 2009; Morse, Kendell y Barton, 2005; entre otros). De los puntos de corte originales de Zigmond y Snaith (1983) se pasa a mostrar otros diferentes que podrían explicarse por las diferencias en los instrumentos elegidos como criterio (gold-standard), las traducciones, el contexto de enfermedad elegido, o la población incluyendo rangos de edad o género (Hinz y Brähler, 2011). También la estructura factorial del instrumento muestra resultados que oscilan entre dos factores (Mykletun, Stordal y Dahl, 2001; Soriano y Salavert, 1996; Spinhoven et al., 1997; entre otros), tres (Dunbar, Ford, Hunt y Der, 2000; Caci et al., 2003; Hunt-Shanks, Blanchard, Reid, Fortier y Cappelli, 2010; Martin et al., 2008; Vallejo, Rivera, Esteve-Vives y Rodríguez-Muñoz, 2012; entre otros) o cuatro (Anderson, 1993 citado en Bjelland et al., 2002; entre otros). Incluso se ha sugerido un único factor latente (Cosco, Doyle, Watson, Ward y McGee, 2012; Kendel et al., 2010; Schonberger y Ponsford, 2010). Ante esta situación, algunos autores en posiciones más extremas apuntan abandonar el estudio de la escala por su inconsistencia (Coyne y Van Sonderen, 2012). Sugieren que la estructura latente de la HAD no es clara y depende de los métodos estadísticos utilizados, observándose cierta incapacidad para diferenciar entre los constructos de ansiedad y depresión, algo que también apunta Cosco et al. (2012). Respecto a la exclusión de ítems somáticos (trastornos del sueño y el apetito) se señala que no se basa en criterios clínicos confirmados (Coyne y Van Sonderen, 2012; Mitchell, Meader, y Symonds, 2010). Así, la subescala de depresión describiría mejor anhedonia (Coyne y Van Sonderen, 2012; Dunbar et al., 2000), y la de ansiedad, impulsividad y/o agitamiento (Caci et al., 2003) o quizá, afectividad negativa (Dunbar et al., 2000). Otros comentarios apuntan que, aunque el HAD funciona como herramienta de screening, quizá no es tan eficaz en la detección de casos (Mitchell et al., 2010) si atendemos a la alta inter-correlación entre subescalas (Bjelland et al., 2002). A pesar de todo esto, el amplio uso clínico del HAD (Keedwell y Snaith, 1996), las buenas propiedades de consis- tencia interna y el apoyo a su estructura bifactorial (Norton et al.., 2012), así como la recomendación de la escala por la Sociedad Española de Reumatología (SER) (Rivera et al., 2006), avalan su administración como instrumento de referencia.

Centrándonos en el objetivo de este trabajo, los estudios realizados con el HAD en muestra española han validado y/o comprobado sus propiedades psicométricas en numerosos estudios con sujetos sanos (Terol et al., 2007), enfermos físicos (Cabrera, Terol, Pastor y Núñez, 2012; Costa, Pérez, Salamero y Gil, 2009; Forjaz, Rodríguez-Blázquez y Martínez-Martín, 2009; López-Roig et al., 2000; Martínez-López et al., 2012; Monsalve, Soriano e Ibáñez, 2001; RodríguezBlázquez et al., 2009; Vallejo et al., 2012) y mentales (Chivite, Martínez, Pérez y Peralta, 2007; De las Cuevas et al., 1995; Herrero et al., 2003; Ibáñez y Caro, 1992; Quintana et al., 2003; Tejero et al., 1986). En general, estos trabajos tratan de comprobar la estructura bifactorial del HAD (Costa et al., 2009; Herrero et al., 2003; López-Roig et al., 2000, Terol et al., 2007; entre otros) y sus puntos de corte (Cabrera et al., 2012; Costa et al., 2009; Herrero et al., 2003; López-Roig et al., 2000; Martínez-López et al., 2012; Terol et al., 2007) entre otras propiedades psicométricas. Estos estudios apoyan el uso del HAD, pero al mismo tiempo se plantea la necesidad de establecer líneas de actuación para la aplicación y establecimiento de criterios o puntos de corte clínicos (Terol, Sanz y Cabrera, 2010). Por esta razón, este trabajo revisa dichos estudios en las diferentes muestras de población española, con el objetivo último de poder integrar directrices de evaluación e interpretación de sus puntuaciones según el colectivo al que se administra.

\section{Método}

\section{Procedimiento}

Se realizó una revisión bibliográfica utilizando las bases de datos scienceDirect, Medline, Psycinfo (WOK), ProQuest, Psicodoc e ISOC sin acotar periodos. Los descriptores fueron: HADS and/or HAD and/or Escala de ansiedad y depresión hospitalaria and/or Hospital Anxiety and depression Scale and/or Validity and/or reliability. Los campos de búsqueda fueron título, palabras clave y abstract. Se encontraron 2384 artículos. Sobre esta primera búsqueda, una vez excluidos coincidentes, duplicados y añadiendo y cruzando con los anteriores el descriptor Spanish and/or muestra española se seleccionaron 18 artículos. Se descartaron aquellos que no evaluaban las propiedades del HAD y/o se limitaban a evaluar ansiedad y depresión. Así, quedaron fuera los de Costa et al. (2009), Noguera et al. (2009) y Soriano y Salavert (1996), obteniendo un total de 15 trabajos $(\boldsymbol{N}=\mathbf{1 5})$. Se siguió un protocolo de revisión que incluye descripción del título y autores; objetivos del estudio; metodología de evaluación (tamaño de la muestra, población sobre la que se realizó el estudio, perfil de los sujetos, instrumentos de evaluación y procedimiento); síntesis de análisis realizados (estructura fac- 
torial, consistencia interna, validez de criterio, inclusión de criterios diagnósticos, sensibilidad y especificidad, puntos de corte), exposición de resultados y discusión de los autores para la elaboración de conclusiones del trabajo.

\section{Resultados}

\section{Características de los estudios revisados}

Cuatro trabajos se han realizado sobre muestras psiquiátricas y/o trastornos emocionales y tres en sujetos sanos. La mayoría de trabajos se han realizado con enfermos físicos, centrándose tres de ellos en pacientes oncológicos, dos con enfermas de Fibromialgia y dos con enfermedad de Parkinson. Finalmente, dos trabajos se llevan a cabo con pacientes de patologías crónicas, entre ellas, dolor crónico (ver Tabla 1). La mayoría de estos trabajos presentan muestras de estudio mayores o igual a 300 sujetos participantes. Uno de los trabajos con pacientes oncológicos se ha realizado con 234 pacientes y uno de los de Fibromialgia con 131. El resto de trabajos presentan un número de sujetos participantes menor a 100 y se corresponden con pacientes con enfermedad mental. En diez de ellos el rango de la edad media oscila entre 48.7 y 66 años, en otros cuatro trabajos la edad media oscila entre 37.1-38.4 años y finalmente, el trabajo realizado con adultos y estudiantes sanos donde la media es de 49.5 y de 21.2 años, respectivamente.

La revisión de estos trabajos muestra, por un lado, el interés y la utilidad del HAD como cuestionario para evaluar ansiedad y depresión en un amplio rango de muestras y, por otro, la diversidad y peculiaridades en cuanto a metodología, procedimiento, análisis y propiedades psicométricas evaluadas, así como qué datos son relevantes para la comprensión y mejora de su aplicabilidad y uso. Tanto es así que, si bien en todos los estudios que presentamos, exceptuando Ibáñez y Caro (1992), se muestra el análisis de la consistencia interna de la escala, no todos ellos lo completan con otras propiedades de validez y/o características del cuestionario (sensibilidad, especificidad). En el caso de la validez de contenido, 13 estudios presentan resultados de los análisis factoriales realizados con las subescalas del HAD. En siete de los 15 trabajos se presentan datos acerca de los puntos de corte, sensibilidad y especificidad del HAD y otros seis abundan en las propiedades de validez concurrente y/o predictiva. En conjunto, cuatro versiones de la escala son utilizadas (Tejero et al., 1986; Ibáñez y Caro, 1992; López-Roig et al., 2000; Herrero et al., 2003). En resumen, de entre los estudios revisados destacan tres de ellos al mostrar con mayor detalle resultados de consistencia, puntos de corte, sensibilidad, especificidad y validez concurrente y predictiva (ver Tabla 1).

Tabla 1. Características de los estudios revisados. Comparativa de análisis realizados.

\begin{tabular}{|c|c|c|c|c|c|c|c|c|}
\hline Estudio, año & Población & $n$ & $\begin{array}{l}\text { Edad } \\
\text { Media }\end{array}$ & $\begin{array}{c}\text { Consistencia } \\
\text { interna }\end{array}$ & $\begin{array}{l}\text { Puntos } \\
\text { de corte }\end{array}$ & $\begin{array}{l}\text { Sensibilidad } \\
\text { especificidad }\end{array}$ & $\begin{array}{l}\text { Análisis } \\
\text { factorial }\end{array}$ & Validez \\
\hline Chivite et al., 2007 (-) & Trastornos emocionales & 97 & 52 & $\sqrt{ }$ & $\sqrt{ }$ & $\sqrt{ }$ & $\sqrt{ }$ & $\sqrt{ }$ \\
\hline Tejero et al., 1986 (1) & Psiquíatricos & 66 & - & $\sqrt{ }$ & & & & $\sqrt{ }$ \\
\hline De las Cuevas et al., $1995(-)$ & Psiquiátricos & 51 & 38.4 & $\sqrt{ }$ & & & & \\
\hline Ibáñez y Caro, 1992 (2) & Psiquíatricos / Sanos & 581 & 37.1 & & & & $\sqrt{ }$ & \\
\hline Terol et al., 2007 (3) & $\begin{array}{l}\text { Sanos } \\
\text { (Adultos y estudiantes) }\end{array}$ & 491 & $\begin{array}{l}\text { Ad:49.5 } \\
\text { Est:21.2 }\end{array}$ & $\sqrt{ }$ & $\sqrt{ }$ & $\sqrt{ }$ & $\sqrt{ }$ & $\sqrt{ }$ \\
\hline Quintana et al., 2003 (1) & Crónicos / Sanos & 685 & 37.7 & $\sqrt{ }$ & & & $\sqrt{ }$ & $\sqrt{ }$ \\
\hline Herrero et al., 2003 (4) & Físicos & 385 & 38.4 & $\sqrt{ }$ & $\sqrt{ }$ & $\sqrt{ }$ & $\sqrt{ }$ & $\sqrt{ }$ \\
\hline Martínez-López et al., 2012 (2) & Oncología & 892 & 57 & $\sqrt{ }$ & $\sqrt{ }$ & $\sqrt{ }$ & $\sqrt{ }$ & \\
\hline López-Roig et al., 2000 (3) & Oncología & 484 & 55.7 & $\sqrt{ }$ & $\sqrt{ }$ & $\sqrt{ }$ & $\sqrt{ }$ & $\sqrt{ }$ \\
\hline Costa et al., 2009 (3) & Oncología & 234 & 55.2 & $\sqrt{ }$ & $\sqrt{ }$ & $\sqrt{ }$ & $\sqrt{ }$ & $\sqrt{ }$ \\
\hline Forjaz et al., 2009 (4) & Parkinson & 387 & 65.9 & $\sqrt{ }$ & & & $\sqrt{ }$ & \\
\hline Rodriguez-Blazquez et al., 2009 (4) & Parkinson & 387 & 65.9 & $\sqrt{ }$ & & & $\sqrt{ }$ & \\
\hline Monsalve et al., 2001 (2) & Ambulatorios dolor crónico & 427 & 53.6 & $\sqrt{ }$ & & & $\sqrt{ }$ & \\
\hline Vallejo et al., 2012 (1) & Fibromialgia & 301 & 48.7 & $\sqrt{ }$ & & & $\sqrt{ }$ & \\
\hline Cabrera et al., 2012 (3) & Fibromialgia & 131 & 50.1 & $\sqrt{ }$ & $\sqrt{ }$ & $\sqrt{ }$ & $\sqrt{ }$ & $\sqrt{ }$ \\
\hline
\end{tabular}

(-) No informa; (1): Versión escala Tejero, 1986; (2): Versión escala Ibáñez y Caro, 1992; (3): Versión escala López-Roig, 2000; (4): Versión escala Herrero, 2003; Ad: Adultos; Est: Estudiantes; $n$ : muestra de población evaluada.

\section{Consistencia interna}

En general, en los trabajos revisados los niveles de consistencia interna son óptimos tanto en la subescala de ansiedad como en la de depresión (ver Tabla 2). Todos los estudios obtuvieron índices superiores a .80 y de hasta .86 (Quintana et al., 2003) en la subescala de ansiedad exceptuando un estudio con sanos (Terol et al., 2007) y pacientes de dolor crónico en régimen ambulatorio (Monsalve et al., 2001). Similares resultados encontramos en la subescala de depresión con rangos de consistencia entre .80 y .87 , salvo en el estudio de Terol et al. (2007) de .71 con pacientes sanos. Sólo cuatro trabajos realizados con enfermos físicos, pacientes con trastornos emocionales y enfermos de Parkinson presentan índices de consistencia de la escala total de ansiedad y depresión con un rango entre .87 y .90 (Chivite et al., 2007; Forjaz et al., 2009; Herrero et al., 2003; MartínezLópez et al., 2012).

Respecto a las correlaciones ítem-test, éstas son aceptables o muy aceptables (ver Tabla 2). En la subescala de an- 
siedad, en cuatro estudios el ítem de menor correlación es el 7 ("Soy capaz de permanecer sentado/ a tranquilo/ a y relajadamente") con coeficientes entre .36 y .60. En cinco estudios es el ítem 13 ("Experimento de repente sensaciones de gran angustia o temor") el de mayor coeficiente de correlación oscilando entre $.57 \mathrm{y}$ .80. Para la subescala de depresión, es el ítem 8 ("Me siento lento $y$ torpe"), el que menor coeficiente muestra en seis estudios con variabilidad entre .25 y .58. En el otro extremo, los ítems 4 ("Soy capaz de reírme y ver el lado gracioso de las cosas") y
12 ("Tengo ilusión por las cosas") en tres estudios aportan el mayor coeficiente de correlación. Para el ítem 4 su variabilidad oscila entre .71 y .79 y el ítem 12 entre .54 y .78. Estos trabajos no mencionan los cambios en la consistencia de la escala al eliminar los ítems de menor correlación, salvo el de López-Roig et al. (2000) en el que no existen cambios y el de Monsalve et al., (2001), en el que aumenta eliminando el ítem 8 ("me siento lento") de la subescala de depresión.

Tabla 2. Consistencia interna y comparativa correlación ítems-test.

\begin{tabular}{|c|c|c|c|c|c|c|c|}
\hline & \multirow[t]{2}{*}{$\alpha$ HADA } & \multirow[t]{2}{*}{$\alpha$ HADD } & \multirow[t]{2}{*}{$\alpha$ HADT } & \multicolumn{2}{|c|}{ Subescala Ansiedad (HADA) } & \multicolumn{2}{|c|}{ Subescala Depresión (HADD) } \\
\hline & & & & Inferior & Superior & Inferior & Superior \\
\hline Tejero et al., 1986 & .81 & .82 & - & - & - & - & - \\
\hline Ibáñez y Caro, 1992 & - & - & - & - & - & - & - \\
\hline De las Cuevas et al., $1995+$ & - & - & - & $.36(7)$ & $.64(13)$ & $.42(10)$ & $.71(4)$ \\
\hline López-Roig, et al., 2000 & .85 & .87 & - & .63 & .79 & .64 & .82 \\
\hline Monsalve et al., 2001 & .74 & .80 & - & - & - & $-(8)$ & - \\
\hline Herrero et al., 2003 & .85 & .84 & .90 & $.60(7)$ & $.80(13)$ & $.58(8)$ & $.79(4)$ \\
\hline Quintana et al., 2003 & .86 & .86 & - & $.46(9)$ & $.72(1)$ & $.47(8)$ & $.76(4)$ \\
\hline Terol et al., 2007 (Adultos) & .77 & .71 & - & $.42(7)$ & $.63(9)$ & $.32(10)$ & $.63(12)$ \\
\hline Terol et al., 2007 (Estudiantes) & .77 & .71 & - & $.37(3)$ & $.57(13)$ & $.25(8)$ & $.54(12)$ \\
\hline Chivite et al., 2007 & .82 & .84 & .87 & $M=.39$ & $M=.39$ & $M=.42$ & $M=.42$ \\
\hline Costa et al., 2009 & .82 & .84 & - & - & - & - & - \\
\hline Forjaz et al., 2009 & $.80^{*}$ & - & $.87 *$ & - & - & - & - \\
\hline Rodriguez-Blazquez et al., 2009 & .81 & .83 & - & $.46(7)$ & $.64(13)$ & $.39(8)$ & $.72(2)$ \\
\hline Vallejo et al., 2012 & .83 & .87 & - & $* *$ & $* *$ & $* *$ & $* *$ \\
\hline Martínez-López et al., 2012 & .83 & .83 & .89 & - & - & - & - \\
\hline Cabrera et al., 2012 & .80 & .85 & - & $.46(11)$ & $.63(13)$ & $.44(8)$ & $.78(12)$ \\
\hline
\end{tabular}

$\alpha:$ Alfa de Cronbach; -No informa; + Coeficiente de Correlación de Spearman; * Indice separación persona; ** Correlaciones significativas, según información de los autores; $O$ Entre paréntesis ítem al que corresponde la correlación; HADA: Subescala de Ansiedad; HADD: Subescala de Depresión: HADT: Escala HADS; $M$ :sólo informa de la media del estudio.

\section{Estructura factorial}

De los trabajos revisados, 13 muestran los resultados de la estructura factorial del HAD y nueve de ellos aportan datos acerca de la varianza explicada (ver Tabla 3). Un gran número de estudios utilizan el método exploratorio de componentes principales para hallar la estructura. Aunque la mayoría de estos trabajos presentan dos factores, en cuatro estudios se muestran posibles soluciones de tres factores (Chivite et al., 2007; Martínez-López et al., 2012; Monsalve et al., 2001; Vallejo et al., 2012). En el primer trabajo (Chivite et al., 2007), no se detallan los ítems incluidos en el denominado "tercer factor" y, finalmente, se presenta la solución clásica de dos factores con los ítems habituales. En el siguiente trabajo (Monsalve et al., 2001), de los tres factores que se obtienen, uno es de depresión y dos de ansiedad. MartínezLópez et al., (2012) muestra el modelo clásico de Zigmond y Snaith y el de tres factores jerárquico concluyendo que éste último se ajustaría mejor. El último de estos trabajos (Vallejo et al., 2012) con tres factores, incluye un factor denominado "ansiedad" con la mayoría de ítems clásicos originales de la subescala $(1,3,5,9$ y 13), un segundo factor llamado "agitación” que engloba los ítems 7, 11 y 14, y en el último factor, denominado "depresión" incluye el resto de ítems de la subescala de depresión (2, 4, 6, 8, 10 y 12). Respecto de la varianza total explicada por los factores, varía entre $46.1 \%$ (Terol et al., 2007) y 59.5\% (Chivite et al., 2007). El estudio de Monsalve et al., (2001) incluye varianza explicada de $51.4 \%$ para los tres factores (ver Tabla 3). 
Tabla 3. Estructura factorial.

\begin{tabular}{|c|c|c|c|c|}
\hline & Método factorial utilizado & $\mathrm{N}^{\circ}$ factores & Factor más explicativo & Varianza explicada \\
\hline Tejero et al., 1986 & - & - & - & - \\
\hline Forjaz et al., 2009 & $A R$ & 1 & - & - \\
\hline De las Cuevas et al., 1995 & AFE & - & - & - \\
\hline Ibáñez y Caro, 1992 & AFE-MCP & 2 & - & - \\
\hline López-Roig et al., 2000 & AFE-MCP & 2 & $\mathrm{D}$ & $55.4 \%$ \\
\hline Herrero et al., 2003 & AFE-MCP & 2 & A & $53.2 \%$ \\
\hline Quintana et al., 2003 & AFE-MCP & 2 & A & $55.4 \%$ \\
\hline Terol et al., 2007 (Adultos) & AFE-MCP & 2 & A & $46.1 \%$ \\
\hline Terol et al., 2007 (Estudiantes) & AFE-MCP & 2 & $\mathrm{D}$ & $40.3 \%$ \\
\hline Costa et al., 2009 & AFE-MCP & 2 & $\mathrm{D}$ & $51.9 \%$ \\
\hline Rodriguez-Blazquez et al., 2009 & AFE-MCP & 2 & A & $49.8 \%$ \\
\hline Cabrera et al., 2012 & AFC & 2 & $\mathrm{D}$ & $51.7 \%$ \\
\hline Chivite et al., 2007 & $\begin{array}{c}A F E-M C P \\
A F C\end{array}$ & $2-3$ & $\mathrm{D}$ & $59.5 \%$ \\
\hline Monsalve et al., 2001 & $\begin{array}{c}A F E-M C P \\
A F C\end{array}$ & $2-3$ & $\mathrm{D}$ & $51.4 \%$ \\
\hline Martínez-López et al., 2012 & $A F C$ & $2-3$ & - & - \\
\hline Vallejo et al., 2012 & $A F C$ & 3 & - & - \\
\hline
\end{tabular}

-: No informan; AFE: Análisis Factorial Exploratorio; AFC: Análisis Factorial Confirmatorio; A: Factor Ansiedad explica más varianza; AR: Análisis de Rasgos; D: Factor Depresión explica más varianza; MCP: Método de Componentes Principales.

En los estudios de estructura bifactorial, y respecto a la subescala de ansiedad (ver Tabla 4), los ítems de menor carga son: a) ítem 3, que en cinco estudios se muestra como el ítem con menor carga (Cabrera et al., 2012; Chivite et al., 2007; Herrero et al., 2003; Quintana et al., 2003; Terol et al., 2007), con cargas que oscilan entre .350 (Terol et al., 2007) y .743 (Ibáñez y Caro, 1992) y b) ítem 7, siendo en tres estudios el ítem con menor carga (Costa et al., 2009; López-Roig et al., 2000; Monsalve et al., 2001). Sus cargas oscilan entre .488 (Monsalve et al., 2001) y .804 (Chivite et al., 2007). Los ítems de mayor carga son a) ítem 1 , en tres estudios ítem más robusto (Chivite et al., 2007; López-Roig et al., 2000; Quintana et al., 2003). Sus cargas oscilan entre .625 (Monsalve et al., 2001) y .825 (Chivite et al., 2007), b) ítem 11, en cuatro estudios ítem con más carga (Cabrera et al., 2012; Herrero et al., 2003; Terol et al., 2007). Sus cargas oscilan entre .642 (Monsalve et al., 2001) y .780 (Quintana et al., 2003) siendo éste último de los más consistentes y menor variabilidad de carga entre estudios (Ver Tabla 4).

Tabla 4. Cargas factoriales. Comparativa y valoración de ítems.

Subescala Ansiedad

3. Siento una especie de temor como si algo malo fuera a suceder

7. Soy capaz de permanecer sentado/a tranquilo/a y relajadamente

5. Tengo la cabeza llena de preocupaciones

13. Experimento de repente sensaciones de gran angustia o temor

1. Me siento Tenso/a o Nervioso/a

11. Me siento inquieto/a como si no pudiera parar de moverme

9. Experimento una desagradable sensación de "nervios y hormigueo en el estómago"

Subescala Depresión

8. Me siento lento/a y torpe

10. He perdido el interés por $\mathrm{mi}$ aspecto personal

14. Soy capaz de disfrutar con un buen libro o un buen programa de radio o TV

2. Sigo disfrutando con las mismas cosas de siempre

6. Me siento alegre

4. Soy capaz de reírme y ver el lado gracioso de las cosas

12. Tengo ilusión por las cosas

1 estudio teniendo carga anómala; ${ }^{*} 1$ estudio siendo ítem menos carga

En la subescala de depresión (ver Tabla 4), destacamos como ítems de menos carga factorial los ítems: a) 8, que en

$\begin{array}{cccc}\mathrm{N}^{\mathrm{e}} \text { estudios } & \mathrm{N}^{\circ} \text { estudios } & \mathrm{N}^{\mathrm{a}} \text { estudios con carga anó- } & \text { Valoración } \\ \text { siendo ítem } & \text { siendo ítem } & \text { mala }(>.40) \text { en el factor al } & \text { negativa del } \\ \text { menor carga } & \text { mayor carga } & \text { que no se adscribe } & \text { ítem }\end{array}$
menor carga mayor carga que no se adscribe ítem

\begin{tabular}{|c|c|c|c|}
\hline 6 & 1 & 2 & $* * * * * * \infty$ \\
\hline 3 & - & 2 & $* * * \circ$ \\
\hline 1 & - & 4 & $*$ oоoо \\
\hline - & 2 & 3 & ооо \\
\hline - & 3 & 2 & оo \\
\hline - & 4 & 1 & 。 \\
\hline - & - & - & \\
\hline 6 & - & 1 & $* * * * * * \circ$ \\
\hline 3 & - & 1 & $* * * \circ$ \\
\hline 1 & - & 4 & * \\
\hline - & - & 2 & оo \\
\hline - & 1 & 2 & оo \\
\hline - & 4 & 1 & 。 \\
\hline- & 5 & 1 & 。 \\
\hline
\end{tabular}

seis estudios se muestra como el ítem con menos carga del factor (Cabrera et al., 2012; Chivite et al., 2007; López-Roig 
et al., 2000; Monsalve et al., 2001; Quintana et al., 2003; Terol et al., 2007). Sus cargas oscilan entre .298 (Monsalve et al., 2001) y .690 (Ibáñez y Caro, 1992) y b) 10, que lo es en tres estudios (Costa et al., 2009; Herrero et al., 2003; Terol et al., 2007). Sus cargas oscilan entre .230 (Terol et al., 2007) y .719 (Ibáñez y Caro, 1992). Además estos ítems son los más variables entre estudios. Como ítems con mayor carga están: a) 4, que en cuatro estudios aparece como mejor carga (Chivite et al., 2007; Herrero et al., 2003; Monsalve et al., 2001; Quintana et al., 2003). Sus cargas oscilan entre .700 (Costa et al., 2009) y .850 (Quintana et al., 2003) y b) 12, que lo es en cinco estudios (Cabrera et al., 2012; Costa et al., 2009; López-Roig et al., 2000), con cargas oscilando entre .593 (Monsalve et al., 2001) y .844 (Cabrera et al., 2012). Son éstos dos ítems los más consistentes entre estudios. (Ver Tabla 4).

\section{Puntos de corte. Sensibilidad y especificidad}

Para este tipo de análisis, entre los instrumentos utilizados como criterio se han utilizado: a) Entrevista Clínica Estructurada para los Trastornos del Eje-I del DSM-IV (SCIDI-Structured Clinical Interview for DSM-IV Axis I Disorders, First, Spitzer, Williams et al., 1996), b) Escala de depresión de Zung (SDS-Self-Rating Depression Scale, Zung, 1965), c) Escala de Evaluación de la depresión de Hamilton (HRSD-Hamilton Rating Scale for Depression, Hamilton, 1967), d) Inventario de depresión de Beck (BDI-Beck Depression Inventory, Beck, 1965), e) Cuestionario de Ansiedad Estado-Rasgo (STAIState Trait Anxiety Inventary, Spielberger, 1971), f) Inventario de Situaciones y Respuestas de Ansiedad (ISRA, Tobal y Cano Vindel, 1986, 1988, 1994) y g) Brief Symptom Inventory (Derogatis, 2000). De los trabajos revisados, en siete de ellos se presentan análisis de sensibilidad y especificidad y puntos de corte tanto para la escala de ansiedad y depresión. Para la escala total se halla en cuatro trabajos. Los puntos de corte para la escala total oscilan en un rango de $10+$ a $16+$ en muestras físicas (Costa et al., 2009; Herrero et al., 2003; MartínezLópez et al., 2012) de 13+ a 17+ para trastornos mentales o 12+ a 15+ para sanos (Chivite et al., 2007; Terol et al., 2007), con sensibilidad y especificidad superior al $70 \%$ excepto la especificidad de las muestras de depresión mayor y trastornos adaptativos de Chivite et al., (2007) y estudiantes de Terol et al., (2007). En la subescala de ansiedad los puntos de corte oscilan entre 8+ y 9+ (Costa et al., 2009; Herrero et al., 2003; López-Roig et al., 2000) y 9+ y 10+ en un estudio (Terol et al., 2007). El trabajo de Cabrera et al., (2012) presenta un punto de corte de más elevado $(12+)$ y el de Chivite et al. (2007) de 7+ y $8+$. Sólo éste último presenta sensibilidad y especificidad inferior al $70 \%$ en una de sus muestras. Para la subescala de depresión, los puntos de corte para la subescala de depresión incluyen un amplio rango de $3+$ a 7+ (Costa et al., 2009; Chivite et al., 2007; Herrero et al., 2003; López-Roig et al., 2000; Terol et al., 2007) y de $10+$ en el estudio de Cabrera et al., (2012). Tanto los índices de sensibilidad como los de especificidad son superiores al $70 \%$ excepto la especificidad de los estudios de Costa et al.,
(2009), Chivite et al., (2007) y el grupo de estudiantes de Terol et al., (2007). En resumen, los puntos de corte para la escala total del HAD oscilan entre $10+-17+$, para la subescala de ansiedad entre 7+-10+, y para la subescala de depresión entre $3+-8+$, exceptuando el estudio con población fibromiálgica (Cabrera et al., 2012). Respecto de las muestras con las que se ha trabajado para comprobar la utilidad del HAD como instrumento de screening, en dos trabajos con pacientes de oncología se presentan puntos de corte similares 8+-9+ para ansiedad y 3+-4+ para depresión (Costa et al., 2009; López-Roig et al., 2000). En otro trabajo con enfermos físicos el punto de corte es de $8+$ para ansiedad y de $5+$ para depresión (Herrero et al., 2003). Los propuestos para pacientes con trastornos emocionales de Chivite et al. (2007): 7+$8+$ para ansiedad y de $6+-7+$ para depresión. Por su parte, son los puntos de corte de pacientes con fibromialgia y otro estudio con muestras sanas los más elevados (Cabrera et al., 2012; Terol et al., 2007). (Ver Tabla 5).

\section{Discusión}

El objetivo de este trabajo pretende poner en común los resultados encontrados con el HAD en diferentes muestras de población española para integrar directrices de evaluación e interpretación de sus puntuaciones según el colectivo al que se administra. En este sentido, esta revisión constataría que el HAD es una escala que utilizada en distintas poblaciones (enfermos físicos, psiquiátricos, personas sanas), obtiene propiedades psicométricas semejantes. El número de estudios encontrados $(N=15)$, y las diferentes poblaciones de estudio evaluadas han permitido comprobar que el HAD funciona bien como instrumento de screening, para evaluar ansiedad y depresión y también como escala total de distrés. Diferentes autores apuntan incluso que la escala de distrés ejecuta mejor que las subescalas por separado (Brennan et al., 2009; Cosco, Doyle, Watson, Ward, y McGee, 2012; Herrmann, 1997; Norton, Cosco, Doyle, Done y Sacker, 2012; Terol et al., 2007; entre otros). En este sentido, se señala que las subescalas podrían medir diferentes aspectos de los trastornos afectivos o tal vez compartir constructos como anhedonia, afectividad negativa o inquietud (Caci et al., 2003; Coyne y Van Sonderen, 2012; Dunbar et al., 2000; entre otros), más que ansiedad o depresión. Así, respecto de la alta correlación entre ambas subescalas sugieren que los ítems no diferencian en gran medida estos dos constructos (Bjelland et al., 2002).

Respecto a la estructura factorial existe cierta homogeneidad en los análisis factoriales realizados y la gran mayoría de los estudios se inclinan por los análisis exploratorios, obteniendo en casi todos los casos dos factores. Los resultados de nuestra revisión coinciden con las revisiones de Bjelland et al., (2002) y Herrmann (1997), al hallar estructura bifactorial en la mayoría de las investigaciones detalladas. Sólo cinco de los estudios realizan análisis confirmatorio (Cabrera et al., 2012; Chivite et al., 2007; Martínez-López et al., 2012; 
Monsalve et al., 2001; Vallejo et al., 2012), encontrando el mejor ajuste de los datos a modelos de dos-tres factores. La diferencia entre el análisis exploratorio y el confirmatorio sería que el último identifica ítems de baja calidad, consiguien- do mejor evidencia de la estructura factorial (Cosco et al., 2011). Es reseñable que sólo uno de los estudios (Forjaz et al., 2009) realiza análisis de rasgos, defendiendo la unidimensionalidad de la prueba.

Tabla 5. Puntos de corte. Sensibilidad y especificidad.

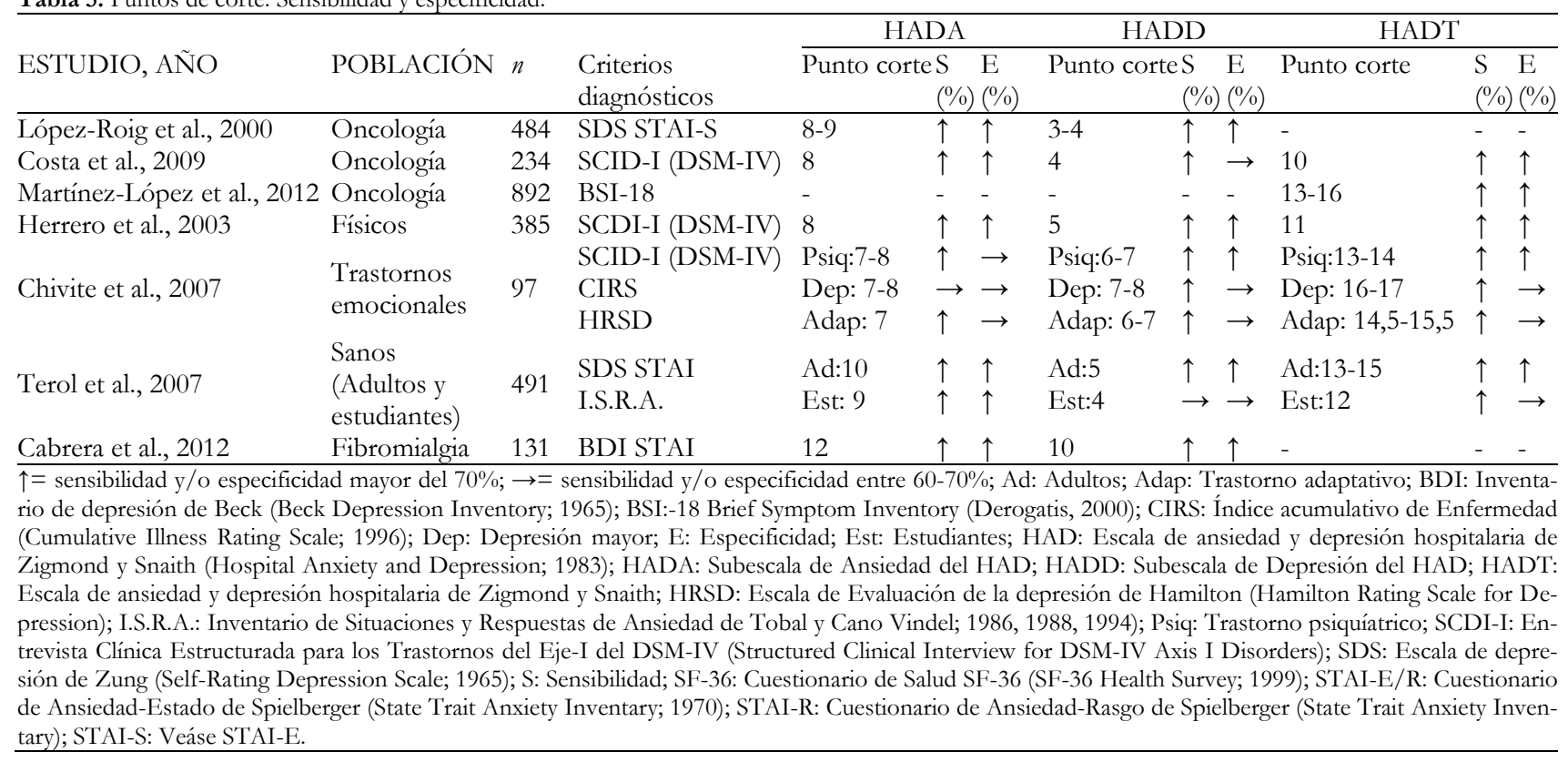

A simple vista, resulta curioso que la estructura de factores coincida según la técnica estadística utilizada, lo que concuerda con la propuesta de Hendrik, van der Ark y Sijtsma, (2013) de que el método psicométrico y las elecciones del investigador pueden ser responsables de la diferente dimensionalidad del HAD. Cabe recordar que en los métodos exploratorios basados en valores Eigen (Kaiser, Scree plots) se compromete la solución al forzar factores (Mykletun et al., 2001). De la misma forma, los análisis exploratorios suelen asumir el número de factores propuestos por los autores originales en la escala. En este sentido, un indicador usualmente utilizado es la carga "anómala" (>.40) o elevada carga de ciertos ítems en el factor al que no se adscribe (o en el que menos satura). Esta carga "anormal" indicaría que algunos ítems están definiendo por igual depresión y ansiedad (ver tabla 4). En nuestra revisión, destaca el estudio de Quintana et al. (2003), donde prácticamente todos los ítems de ambas subescalas contienen carga "anormal" de su otro ítem-factor.

En cuanto a la consistencia interna, existe semejanza en los estudios revisados, con índices alfa de cronbach aceptables o buenos para ambas subescalas, situándose siempre por encima de .70 y en la gran mayoría de estudios por encima de .80 independientemente de la muestra evaluada (físicos, psiquiátricos o sanos). En lo que respecta a las correlaciones ítem-test cuando profundizamos en su variabilidad y comparamos los ítems encontramos cierta consistencia en los de menor y mayor correlación entre estudios. Por esta razón, teniendo en cuenta tanto cargas factoriales, como correlaciones ítem-test, una propuesta sería -suprimiendo los ítems 3 y 7 de la HADA y los ítems 8 y 10 de la HADD- revisar y comprobar una nueva configuración y propiedades del HAD. Estos ítems son los que presentan el siguiente un perfil: a) mayor número de estudios en los que su carga factorial es la más baja o aparece entre las cargas más bajas, b) mayor número de estudios con carga anómala $(>.40)$ del ítem en el factor al que no se adscribe, c) mayor número de estudios en los que el ítem obtiene cargas considerables en ambos factores con pequeña diferencia entre sí, d) mayor número de estudios donde el ítem tiene la menor o una de las menores correlaciones ítem-test. Los ítems propuestos aparecen como más débiles en distintos estudios a lo largo de los años y sobre distintas muestras y ámbitos de evaluación (Ver tabla 4).

Algunos autores discuten la brevedad del HAD frente a otros instrumentos como el Patient Health Questionnaire (PHQ-9, Kroenke, Spitzer y Williams, 2001) o Generalized Anxiety Disorder 7-item (GAD-7, Spitzer, Kroenke, Williams y Löwe, 2006). Esto, unido a la ambigüedad de la estructura factorial del HAD y la parte común que pueden compartir las subescalas del HAD, PHQ-9 y GAD-7, podría apoyar cierta preferencia hacia estos dos últimos en la práctica clínica (Kendel et al., 2010; Norton et al., 2012; Spitzer et al., 2006). Trabajar en el acortamiento del HAD, suprimiendo los ítems mencionados, supondría un camino a seguir si se mantienen las propiedades psicométricas y la estructura fac- 
torial. Hendrik et al., (2013), halla en su análisis de Respuesta al Ítem (modelo probabilístico Mokken) dos subescalas con cinco ítems: HADA (ítems 1, 3, 5, 9 y 13) y HADD (ítems 2, 4, 6, 8 y 12), dejando fuera ítems de pobre calidad como los siguientes: 7, 11 y 10, 12. Otros estudios también proponen ítems con pobres propiedades como son el 7 (Schonberger y Ponsford, 2010), 8 (Forjaz et al., 2009; Monsalve et al., 2001; Rico, Restrepo y Molina, 2006; Schonberger y Ponsford, 2010; entre otros) y 10 (Herrmann, 1997; Kendel et al., 2010; Schonberger y Ponsford, 2010), coincidiendo los de nuestra propuesta, a la que añadimos el ítem 3.

Pero tal vez el dilema más importante en este trabajo se encuentra en la decisión o manejo de los diferentes puntos de corte del HAD según muestra en la que se evalúa. En nuestros resultados, los valores de sensibilidad y especificidad comparados muestran que el HAD funciona bien, por encima siempre del $60 \%$ en ambos indicadores y por encima del $70 \%$ en gran parte de las ocasiones. Pero existe una amplia variabilidad de puntos de corte por estudio, que se mueven en horquillas de $7+-10+/ 12+$ para el HADA, 3+$8+/ 11+$ para el HADD y $10+/ 17+$ para la escala total (HADT). Algunos interrogantes se plantean: ¿Tal vez -como ocurre en el análisis factorial- la metodología empleada y las medidas criterio influyen en los puntos de corte hallados? ¿Debemos usar puntos de corte diferentes como screening en función de la muestra de estudio? Y en caso de grupos de intervención o tratamiento: ¿son útiles los puntos de corte según la población? Esta variabilidad de los puntos de corte no es exclusiva de la muestra española y como citábamos en la introducción varias las revisiones señalan esta cuestión (Bjelland et al., 2002; Brennan et al., 2009; Hinz y Brähler, 2011; Morse et al., 2005).

\section{La evaluación e interpretación del HAD. Futuras lí- neas de investigación}

El amplio uso de los clínicos y su aceptación por parte de los propios pacientes recomienda seguir usando la escala, pero nuestra propuesta sería corroborar el uso de la escala total como herramienta de cribado de distrés psicológico y utilizar las puntuaciones de las subescalas como un valor añadido a la evaluación clínica. Todo ello hasta que la estructura subyacente del HAD y por tanto su capacidad para evaluar ansiedad o depresión por separado se perfeccione. La investigación futura debería concentrarse en determinar si hay que usar puntos de corte independientemente de las poblaciones y no sujeta a la idiosincrasia de la muestra evaluada, utilizando criterios clínicos originales (Zigmond y Snaith, 1983). En estos momentos, el HAD podría parecer un termómetro que requiere distinto indicador de grado según el individuo que se tome la temperatura. Sirva como ejemplo la población de enfermas de fibromialgia (Cabrera et al., 2012). Utilizar el punto de corte obtenido $(12+)$ dejaría fuera de tratamiento a muchos potenciales pacientes, dado que esta población se caracterizaría por tener altos niveles de ansiedad y depresión, y no por ello debemos concentrarnos en los que más afectación muestren por encima del punto de corte. En el otro extremo, la población oncológica (Costa et al., 2009; López-Roig et al., 2000) con puntos de corte bajos podría provocar la atención a numerosos pacientes, con el consiguiente consumo de recursos y pérdida de eficiencia de los mismos. Por otra parte, la multiplicidad de estudios intentando hallar los puntos de corte en cada población, pueden hacer perder la perspectiva sobre la razón de ser de un punto de corte Gold-Standard. Mientras tanto, como apunta Morse et al.. (2005), usar los puntos de corte más bajos con una sensibilidad suficiente para el cribado, y con una especificidad ajustada parece ser lo más coherente. En los puntos de corte medios y superiores derivar al paciente a los servicios de psiquiatría y/o psicología y en cualquier caso contrastar con una prueba clínica diagnóstica. Es preferible sacrificar especificidad de la prueba para conseguir la máxima sensibilidad. Así, lo importante es que ningún paciente afecto quede fuera de evaluación ulterior o más profunda por no llegar a un punto de corte. Además, los instrumentos de screening deben facilitar la labor del clínico, pero nunca sustituirla.

Por otra parte sería deseable contrastar los puntos de corte de cada población en otros contextos y ámbitos sanitarios, como la atención primaria o especializada, contar con grupos de control, muestras mixtas (sanos, físicos, psiquiátricos), y con diferentes características demográficas y clínicas (rangos de edad, patologías, situación de enfermedad, entre otros). Todo ello, buscando la representatividad poblacional y el rigor metodológico necesario para consolidar las diversas aplicaciones clínicas del HAD.

A modo de resumen, coincidiendo con diferentes autores sugerimos que se afiance el trabajo con los puntos de corte para escala total HADT y sugerimos, como ya apuntaron otros estudios, el punto de corte $13+$ para la discriminación de casos (Clover, Carter, Macinnon y Adams, 2009; Hinz y Brähler, 2011; Singer et al., 2009; Terol et al., 2007).

A pesar de mantener la estructura bifactorial del HAD (Norton et al., 2012), apoyada por su amplio uso y el apoyo empírico demostrado, parece interesante abundar en los análisis factoriales confirmatorios y los métodos de Teoría de Respuesta al Ítem, como análisis de rasgos o análisis de escala Mokken (Cosco, Doyle, Ward y McGee, 2011; Emons, Sijtsma y Pedersen, 2012; Hendrik et al., 2013). Asimismo tratar de acortar la escala comprobando de nuevo las propiedades psicométricas del instrumento aportaría brevedad y claridad de la estructura factorial. Un punto de partida podría ser la supresión de los ítems ya mencionados en esta revisión (HADA: 3 y 7; HADD: 8 y 10).

Agradecimientos.-Este estudio forma parte del Programa de Formación de Profesorado Universitario FPU Convocatoria 2010. Ministerio Educación. Referencia AP2010-1870. 


\section{Referencias}

Abiodun, O. A. (1994). A validity study of the Hospital Anxiety and Depression Scale in general hospital units and a community sample in Nigeria. British Journal of Psychiatry, 165, 669-672.

Aylard, P. R., Gooding, J. H.; McKenna, P. J., \& Snaith, R. P (1987). A validation study of three anxiety and depression self-assesment scales. Journal of Psychosomatic Research, 31 (2), 261-268.

Barczack, P., Kane, N., Congdon, A. M., Clay, J.C., Betts, T., et al.. (1988). Patterns of Psychiatric Morbidity in a Genito-Urinary Clinic. A validation of The Hospital Anxiety and Depression Scale. British Journal of Psychiatry, 152, 698-700.

Beck, A. T., Ward, C. H., Mendelson, M., \& Erbaugh, J. (1965). An inventory for measuring depression. Archives General Psychiatry, 4, 561-571

Berard, M. F. R., \& Ahmed N. Mz. (1995). Hospital Anxiety and Depression Scale (HADS) as screening instrument in a depressed adolescent and young adult population. International Journal of Adolescence Medicine and Health, 8(3), 157-166

Bjelland, I., Dahl, A. A., Haug, T. T., \& Neckelmann, D. (2002). The validity of the Hospital Anxiety and Depression Scale. An updated literature review. Journal of Psychosomatic Research, 52, 69-77.

Brennan, C., Worrall-Davies, A., McMillan, D., Gilbody, S. \& House, A. (2010). The Hospital Anxiety and Depression Scale: A diagnostic metaanalysis of case-findig ability. Journal of Psychosomatic Research, 69, 371378.

Burvill, G., Anderson, C. S., Jamrozik, K., Stewart-Wynne, E. G., \& Chakera, T.M.H. (1995). Screening instruments for depression and anxiety following stroke: experience in the Perth community stroke study. Acta Psychiatrica Scandinavica, 91, 252-257

Cabrera-Perona, V., Terol-Cantero, M.C., Núñez Núñez, R., y Pastor Mira, M. A. (2012). Adecuación del HAD para población con Fibromialgia. Póster presentado en el IX Congreso Internacional de la Sociedad Española de Ansiedad y Estrés. Valencia. 2012.

Caci, H., Baylé, F. J., Mattei, V., Dossios, C., Robert, P., \& Boyer, P. (2003). How does the Hospital and Anxiety and Depression Scale measure anxiety and depression in healthy subjects? Psychiatry Research, 118(1), 89-99. Doi:10.1016/S0165-1781(03)00044-1

Chivite, S., Martinez, M., Pérez, E., y Peralta, V. (2007). HADS: Estudio de criterios de validez interna y externa en una muestra española de pacientes hospitalizados. Cuadernos de Medicina Psicosomática y Psiquiatría de Enlace, 84, 9-17.

Clover, K., Carter, G. L., Macinnon, A., \& Adams, C. (2009). Is my patient suffering clinically significant emotional distress? Demonstration of a probabilities approach to evaluating algorithms for screening for distress. Support Care Cancer, 17, 1455-1462.

Constantini, M., Musso, M., \& Viterbori, P. (1999). Detecting psychological distress in cancer patients: validity of the Italian version of the Hospital Anxiety and Depression Scale. Support Care Cancer, 7, 121-127.

Coyne, J. C., \& van Sonderen, E. (2012). No further research needed: Abandoning the Hospital and Anxiety Depression Scale (HADS). Journal of Psychosomatic Research, 72(3), 173-174. Doi:10.1016/j.jpsychores.2011.12.003

Cosco, T., Doyle, F., Ward, M., \& McGee, H. (2011). Latent structure of the hospital anxiety and depression scale: a 10 year systematic review. Psychology Articles. Doi:10.1016/j.jpsychores.2011.06.008

Cosco, T. D., Doyle, F., Watson, R., Ward, M., \& McGee, H. (2012). Mokken scaling analysis of the Hospital Anxiety and Depression Scale in individuals with cardiovascular disease. General Hospital Psychiatry, 34(2), 167-172. Doi:10.1016/j.genhosppsych.2011.11.005

Costa, G., Pérez, X., Salamero, M., y Gil, F. L. (2009). Discriminación del malestar emocional en pacientes oncológicos utilizando la Escala de Ansiedad y Depresión Hospitalaria (HADS).

Costa, G., Martin, X. Perez, Baro, M. Salamero, Moncayo, y Gil, F.L. (2009). Screening distress in cancer patients using the Hospital Anxiety and Depression Scale (HADS). Ansiedad y Estres, 15(2-3), 217-229.

De las Cuevas, C., Garcia-Estrada Perez, A., y González de Rivera, J.L. (1995). Hospital Anxiety and Depresión Scale y Psicopatología Afectiva. Anales de Psiquiatría, 2 (4), 126-130.
Derogatis, L. R. (2000). Brief Symptom Inventory 18. Minneapolis: National Computer Systems Pearson, Inc.

Dunbar, M, Ford, G, Hunt, K, \& Der, G. (2000). A confirmatory factor analisis of the Hospital Anxiety and Depression Scale: comparing empirically and theoretically derived structures. British Journal of Clinical Psychol ogy, 39, 79- 94 .

El-Rufaie, O. E. F., \& Absood, G. H. (1995). Retesting the Validity of the arabic version of the hospital Anxiety and depression (HAD) scale in primary health care. Social Psychiatry and Psychiatric Epidemiology, 30, 26-31

Emons, W. H. M., Sijtsma, K, \& Pedersen S.S. (2012). Dimensionality of the Hospital Anxiety and Depression Scale (HADS) in cardiac patients: comparison of Mokken scale analysis and factor analysis. Assessment, 19 , 337-353. Recuperado de http://dx.doi.org/10.1177/107319111038495.

Forjaz, M. J., Rodriguez-Blazquez, C., \& Martinez-Martin, P., Longitudinal Parkinsons Dis Patien (2009). Rasch Analysis of the Hospital Anxiety and Depression Scale in Parkinson's Disease, Movement Disorders, 24 (4), 526-532.

Fossati, L., \& Marzocchi, P. (2008). Factor analysis of the Hospital Anxiety and Depression Scale: Comparison of factor structures on a sample of Italian patients with cardiological disorder. Giornale di Psicologia, 2(3), 143-150.

Hamilton, M. (1967). Development of a rating scale for primary depressive illness. British Journal of Social and Clinical Psychology, 6, 278-96

Hammer, D., Sanjeev, D., Butterworth, E., \& Barczack, P. (1991). Using the Hospital Anxiety and Depression Scale to Screen for Psychiatric Disorders in People presenting with Deliberate Self-Harm. British Journal of Psychiatry, 158, 782-84.

Hendrik, J., L., van der, A., \& Sijtsma, A. K. (2013). Methodological artifacts in dimensionality assessment of the Hospital Anxiety and Depression Scale (HADS). Journal of Psychosomatic Research, 74, 116-121

Herrero, M. J., Blanch, J., Peri, J. M., De Pablo, L., Pintor, A., \& Bulbena, A. (2003). A validation study of the Hospital anxiety and depression scale (HADS) in a Spanish population. General Hospital Psychiatry, 25, 277-283.

Herrmann, C. (1997). International Experiences with the Hospital Anxiety and Depression Scale-A review of Validation data and Clinical results. Journal of Psychosomatic Research, 1 (42), 17- 41.

Hinz, A., \& Brähler, E. (2011). Normative values for the hospital anxiety and depression scale (HADS) in the general German population. Journal of Psychosomatic Research, 71(2), 74-78. Doi:10.1016/j.jpsychores.2011.01.005

Hunt-Shanks, T., Blanchard, C., Reid, R., Fortier, M., \& Cappelli, M. (2010). A psychometric evaluation of the Hospital Anxiety and Depression Scale in cardiac patients: Addressing factor structure and gender invariance. British Journal of Health Psychology, 15, 97-114.

Ibáñez E. y Caro, I. (1992). La escala Hospitalaria de ansiedad y depresión. Su utilidad práctica en Psicología de la Salud. Boletín de Psicología, 36, 43 69

Keedwell, P. \& Snaith, R. P., (1996). What do anxiety scales measure? Acta Psychiatrica Scandinavica 93, 177-180.

Kendel, F., Wirtz, M., Dunkel, A., Lehmkuhl, E., Hetzer, R., \& RegitzZagrosek, V. (2010). Screening for depression: Rasch analysis of the dimensional structure of the PHQ-9 and the HADS-D. Journal of Affective Disorders, 122(3), 241-246. Doi:10.1016/j.jad.2009.07.004

Kroenke, K., Spitzer, R. L., \& Williams, J. B. (2001). The PHQ-9: validity of a brief depression severity measure. Journal of General Internal Medicine, 16(9), 606- 613

Leung, C. M., Ho, S., Kan, C. S., Hung, C. H., \& Chen, C. N. (1993) Evaluation of the Chinese version of the hospital Anxiety and Depression Scale. A cross cultural perspective. Int J Psychosom, 40, 29-34.

Lewis G., \& Wessley, S. (1990). Comparison of the General Health Questionnaire and The Hospital Anxiety and Depression Scale. British Journal of Psychiatry, 157, 860-864.

López-Roig, S., Terol, M. C., Pastor, M. A., Massutí, B., Rodríguez-Marín, J., Neipp, M. C., Leyda, J.I., Martín-Aragón, M., Sánchez, S., y Sitges, E. (2000): Ansiedad y Depresión. Validación de la escala HAD en pacientes oncológicos. Revista de Psicología de la Salud,2 (12), 127-157. 
Malasi, T. H., Mirza, I. A., \& el Islam, M. F. (1991). Validation of the hospital Anxiety and depression Scale in Arab patients. Acta Psychiatrica Scandinavica, 84, 323-326.

Martínez López, P., Durá Ferrandis, E., Andreu Vaillo, Y., Galdón Garrido M.J., Murgui Pérez, S., \& Ibáñez Guerra, E. (2012). Structural validity and distress screening potential of the Hospital Anxiety and Depression Scale in cancer. International Journal of Clinical and Health Psychology, 12, 435-447

McCue, P., Martin, C. R., Buchanan, T., Rodgers, J., \& Scholey, A. B. (2003). An investigation into the psychometric propierties of the Hospital Anxiety and Depression Scale in individuals with chronic fatigue syndrome. Psychology Health and Medicine, 4 (8)

Miklavcic, I. V., Snoj, Z., Mlakar, J., \& Pregelj, P. (2008). Validation of the Slovenian version of Hospital Anxiety and Depression Scale in female cancer patients.Psycbiatria Danubina, 20(2), 148-152.

Mykletun, A., Stordal, E., \& Dahl, A.A. (2001) Hospital Anxiety and Depression (HAD) scale: factor structure, item analyses and internal consistency in a large population. British Journal of Psychiatry, 179, 540 -544.

Mitchell, A. J., Meader, N., \& Symonds, P. (2010). Diagnostic validity of the Hospital Anxiety and Depression Scale (HADS) in cancer and palliative settings: A meta-analysis. Journal of Affective Disorders, 126, 335-348.

Monsalve, V., Soriano, J., y Ibañez, E. (2001). Fiabilidad y estructura factorial de la escala de ansiedad y depresión hospitalaria (HAD) en una muestra de pacientes con dolor crónico. Boletín de Psicología, 72, 7-21.

Moorey, S., Greer, S., Watson, M., Gorman C., Rowden L., Tunmore, R., et al. (1991). The factor structure and factor stability of the hospital anxiety and depression scale in patients with cancer. British Journal of Psychiatry, 15, 255-259.

Morse, R., Kendell, K., \& Barton, S. (2005). Screening for depression in peoplewith cancer: the accuracy of the hospital anxiety and depression scale.Clin. Effect Nurs. 9, 188-196.

Mumford, D. B., Tareen, I.A., Bajwa, M. A., Bathi, M. R., \& Karin, R. (1991) The translation and evaluation of an Urdu version of the hospital Anxiety and Depression scale. Acta Psychiatr scand, 83, 81-85.

Noguera, A., Centeno, C., Carvajal, A. Portela, M. A., Urdiroz, J., \& Martinez, M. (2009). Spanish "Fine Tuning” of Language To Describe Depression and Anxiety. Journal of Palliative Medicine, 12(8), 707-712.

Norton, S., Cosco, T., Doyle, F., Done, J., \& Sacker, A. (2012). The Hospital Anxiety and Depression Scale: A meta confirmatory factor analysis. Journal of Psychosomatic Research, 74(1), 74-81

Nortvedt, M. W., Riise, T., \& Sanne, B. (2006). Are men more depressed than women in Norway? Validity of the Hospital Anxiety and Depression Scale. Journal of Psychosomatic Research, 60(2), 195-198. Doi:10.1016/j.jpsychores.2005.07.002

Quintana, J. M., Padierna, A., Esteban ,C., Arostegui I., Bilbao, A., \& Ruiz, I. (2003). Evaluation of the Psychometric characteristics of the Spanish version of the Hospital Anxiety and Depression Scale. Acta Psychiatrica Scandinavica, 10, 216-221.

Razavi, D., Delvaux, N., Bredart, A., Paesmans, M., Debuscheer, L., Bron, D., \& Stryckmans, P. (1992). Screening for Psychiatric Disorders in a Lymphoma Outpatient Population. European Journal of Cancer, 28, 86972 .

Rico, J., Restrepo, M., y Molina, M. (2006). Adaptación y validación de la Escala Hospitalaria de Ansiedad y Depresión (HAD) en una muestra de pacientes con cáncer del instituto nacional de cancerología de Colombia. Avances en medición, 3,73-86.

Rivera, J., Alegre C., Ballina F., Carbonell, J., Carmona, L., Castel, B., et al.. (2006). Documento de consenso de la Sociedad Española de Reumatología sobre la fibromialgia. Reumatología Clínica, 2, 55-66.

Rodriguez-Blazquez, C. De Pedro-Cuesta, J., Martínez-Martín, P., \& ELEP Group (2008). Hospital anxiety and depression scale in Parkinson's disease: psychometric attributes in a Spanish collaborative study (ELEP). European Journal Of Neurology, 15, 130-131.

Rodriguez-Blazquez, C., Frades-Payo, B., Forjaz, M.J., de Pedro-Cuesta, J., Martinez-Martin, P., \& Longitudinal Parkinsons Dis Patien Group. (2009). Psychometric Attributes of the Hospital Anxiety and Depression Scale in Parkinson's Disease. Movement Disorders, 24(4), 519-525.
Savard, J., Laberge, B., Gauthier, J.G., Ivers, H., \& Bergerson, M. G. (1998). Evaluating Anxiety and Depression in HIV-Infected patients. Journal of Personality Assessment, 71(3), 349-367.

Schonberger, M., \& Ponsford, J. (2010). The factor structure of the Hospital Anxiety and Depression Scale in individuals with traumatic brain injury. Psychiatry Research, 179, 342-349.

Silverstone, P. H. (1994). Poor Efficay of the Hospital Anxiety and depression scale in the diagnosis of major depressive disorder in both medical and psychiatric patients. Journal of Psychosomatic Research, 5 (38), 441-450.

Singer, S., Kuhnt S., Goäntze, H., Hauss, J., Hinz, A., Liebmann, A., et al.. (2009). Hospital anxiety and depression scale cutoff scores for cancer patients in acute care. British Journal Cancer, 100,908-912

Soriano, J., y Salavert, L. (1996). The Hospital Anxiety and Depression Scale questionnaire in physical danger and evaluation situations. Couns Psychol, 9, 281-96.

Spielberger, C. D., Gorsuch, R. L., \& Lushene, R. E. (1970). Manual for the State-Trait Anxiety Inventory. Palo Alto: Consulting Psychologist Press. (Adaptación española en Editorial TEA.)

Spinhoven, P. H., Ormel, J., Sloekers, P. P., Kemper, G. I., Speckens, A. E., \& Van hemert, A. M. (1997). A validation study of the hospital Anxiety and Depression Scale in different groups of Dutch subjects. Psychol med, 27, 363-370.

Spitzer, R. L., Kroenke, K., Williams, J. B. W., \& Löwe, B. (2006). A brief measure for assessing generalized anxiety disorder: the GAD-7. Archive Internal Medicine 166,1092-1097.

Tejero, A., Guimera, E., Farré, J. M., y Peri, J. M. (1986). Uso clínico del HADS (Hospital Anxiety and Depression Scale) en población psiquiátrica: un estudio de sensibilidad, fiabilidad y validez. Revista del Departamento Psiquiatría Facultad de Medicina U. Barcelona, 12, 233-238.

Terol, M. C., López-Roig, S., Rodríguez-Marín, J., Martín-Aragón, M., Pastor, M. A., y Reig, M. T. (2007). Propiedades psicométricas de la Escala Hospitalaria de Ansiedad y Estrés (HAD) en población española. Ansiedad y Estrés, 13 (2-3), 163-176.

Terol, M. C., Sanz, Y., y Cabrera, V. (2010, Septiembre). Adaptación Española del HAD: Una revisión de estudios. Póster presentado en el VIII Congreso Internacional de la Sociedad Española de Ansiedad y Estrés. Valencia. 2010.

Tobal, J. J. y Cano Vindel, A. (1986). Manual del Inventario de Situaciones y Respuestas de Ansiedad - ISRA-. Madrid: TEA. (2a Ed., 1988; 3a Ed., 1994).

Tyrer, P., \& Methuen, C. (2007). Rating Scales in Psychiatry. London: RCPSYCH Publications.

Untas, A., Aguirrezabal, M., Chauveau, P., Leguen, E., Combe, C., \& Rascle, N. (2009). Anxiety and depression in hemodialysis: validation of the Hospital Anxiety and Depression Scale (HADS). Néphrologie \& Thérapeutique, 5(3), 193-200. Doi:10.1016/j.nephro.2009.01.007

Upadhyaya, A. K., \& Stanley, I. (1997). Detection of depression in primary care: Comparison of two self-administered scales. International Journal of Geriatric Psychiatry, 12, 35-37.

Vallejo, M. A., Rivera, J., Esteve-Vives, J., y Rodríguez-Muñoz, M. F. (2012). Uso del cuestionario Hospital Anxiety and Depression Scale (HADS) para evaluar la ansiedad y la depresión en pacientes con fibromialgia. Revista de Psiquiatría y Salud Mental, 5(2), 107-114.

Visser, M. C., Koudstaal, P.J., Ruud, A. M., Erdamn, J. W., Deckers, J. P., Passchier, J., et al.. (1995). Measuring Quality of life in patients with myocardial infarction or stroke: a feasibility study of four questionnaires in the Netherlands. Journal of Epidemiology and community Health, 49, 513517.

White, D., Leach, C., Sims, R., Atkinson, M., \& Cottrell, D. (1999). Validation of the Hospital Anxiety and Depression Scale for use with adolescents. British Journal of Psychiatry, 175, 452-454.

Zigmond A., \& Snaith, R. (1983). The Hospital Anxiety and Depression Scale. Acta Psychiatrica Scandinavica, 67, 361-370.

Zung, W. W. K. (1968). A Self-Rating Depression Scale. Archives of General Psychiatry, 12, 63-70.

(Artículo recibido: 12-04-2013; revisado: 03-06-2013; aceptado: 25-06-2014) 